\title{
Gallium Ga 68 Citrate
}

National Cancer Institute

\section{Source}

National Cancer Institute. Gallium Ga 68 Citrate. NCI Thesaurus. Code C121554.

A radiopharmaceutical citrate salt form of the positron-emitting radioisotope gallium Ga 68 , with potential imag ing activity upon positron emission tomography (PET). Upon administration of Gallium Ga 68 citrate, the gallium Ga 3+ ion dissociates from the weak citrate chelator. As Ga3+ is very similar to iron ( $\mathrm{Fe} 3+)$ in chemical properties, this ion acts as an iron analogue. Ga3+ binds to the iron-binding protein transferrin, distributes in blood, and enters and accumulates in the extracellular fluid space of inflamed or tumorbearing tissue. In turn, the Ga3+-transferrin complex binds to transferrin receptors and is taken up by tumor cells. Tumor cells can then be imaged upon PET. Compared to healthy cells, tumor cells have increased iron metabolism and transferrin receptor expression. Increased Ga3+ uptake is seen in inflamed and infected tissues as well. 